American Journal of Biotechnology and Biochemistry 3 (2): 87-91, 2007

ISSN 1553-3468

(C) 2007 Science Publications

\title{
Beta-2-Microglobulin in Autism Spectrum Disorders
}

\author{
${ }^{1}$ Paula Goines, ${ }^{1}$ Joseph Schauer, ${ }^{1}$ Luke Heuer, ${ }^{2}$ Paul Ashwood and ${ }^{1}$ Judy Van de Water \\ ${ }^{1}$ Division of Rheumatology, Allergy and Clinical Immunology, University of California at Davis \\ ${ }^{2}$ Department of Medical Microbiology and Immunology and the M.I.N.D. Institute \\ University of California at Davis
}

\begin{abstract}
Autism spectrum disorders (ASD) are heterogeneous neurodevelopmental diseases of unknown etiology. There are no biological markers for ASD and current diagnosis is based on behavioral criteria. Recent data has shown that MHC I, a compound involved in adaptive immune function, is also involved in neurodevelopment, synaptic plasticity and behavior. It has been suggested that altered MHC I expression could play a part in neurodevelopmental diseases like ASD. To address this possibility, we measured plasma levels of beta-2-microglobulin $(B 2 \mathrm{~m})$, a molecule that associates with MHC I and is indicative of MHC I expression, in 36 children with autism, 28 typically developing controls and subjects with developmental disabilities $(\mathrm{n}=16)$ but not autism. The age range of our study population was 17-120 months. We found no statistically significant differences in plasma $\beta 2 \mathrm{~m}$ levels between groups. Therefore, plasma levels of $\beta 2 \mathrm{~m}$ measured in early childhood in autism may not reflect changes in MHC class I in autism.
\end{abstract}

Key words: MHC class I, autism, immune system

\section{INTRODUCTION}

Autism Spectrum Disorders (ASD) are a group of neurodevelopmental diseases characterized by a range of social difficulties, deficits in communication and restricted stereotypic behaviors and interests ${ }^{[1,2]}$. The etiology of ASD is unknown and may involve genetic, environmental and immune factors. There are no known definitive biological markers for ASD or its phenotypic variations and current diagnosis is based on behavioral criteria. In the United States, the incidence of ASD has risen to an estimated 1:150 children $^{[3]}$; a finding which has hastened the search for relevant biological markers associated with these disorders.

Several studies have suggested a potential link between ASD and immune system dysfunction (reviewed in ${ }^{[4]}$. Issues such as increased inflammation in the brain ${ }^{[5,6]}$, autoantibodies to brain tissues (reviewed $\mathrm{in}^{[4,}{ }^{[111]}$ ) and maternal/perinatal infections $\left({ }^{[12,13]}\right.$, reviewed $\left.\mathrm{in}^{[14]}\right)$ have been associated with the development of ASD. Immune system related genes have also been linked to ASD, including the complement component $\mathrm{C} 4 \mathrm{~B}$ gene and the extended HLA haplotype B44-S30-DR4 $\left.{ }^{[15-16]}\right)$. Due to the extensive crosstalk between the immune system and central nervous system (CNS) during development and beyond ${ }^{[17-19]}$, it is conceivable that aberrant immune activity could be detrimental to CNS function.

The major histocompatibility complex (MHC) encodes several genes that play a central role in adaptive immune responses. In addition to its immunological functions, recent evidence suggests that MHC I expressed on neuronal cells may also be important for brain development and function (reviewed in ${ }^{[18,20])}$. It has been shown in several studies that MHC I is crucial for synaptic plasticity in the developing and adult mammalian brain $^{[21-23]}$. Furthermore, it has been suggested that deficiencies in MHC I expression may play a role in neurological diseases including schizophrenia (reviewed $\mathrm{in}^{[24]}$ and autism $^{[18]}$. B-2- microglobulin $(B 2 \mathrm{~m})$ is an essential component of MHC I and can be detected in the serum in lieu of directly measuring cellular expression of MHC I ${ }^{[25]}$. In the current study, we measured levels of $\beta$ $2 \mathrm{~m}$ in the sera of subjects with ASD compared to children with developmental delays but not autism (DD) and typically developing controls from the general population (TD). We further divided the ASD group based on phenotypic differences in the onset of the disease (early onset-EO and regressive) ${ }^{[26]}$, since differing forms of ASD may have distinct biomarkers. We hypothesized that children with autism would have

Corresponding Author: $\quad$ Dr. Judy Van de Water, Division of Rheumatology/Allergy and Clinical Immunology, 451 E. Health Sci. Dr. UC Davis, Davis, CA 95616 
lower plasma levels of $\beta 2 \mathrm{~m}$; a finding which may be responsible for aspects of the disorder.

\section{MATERIALS AND METHODS}

Subjects and samples: This study examined 79 children enrolled through the M.I.N.D. (Medical Investigations of Neurodevelopmental Disorders) Institute Clinic as part of the ongoing CHARGE (Childhood Autism Risk from Genetics and Environment) study at UC Davis ${ }^{[27]}$. The sample population consisted of children with early onset autism (AU-EO; $\mathrm{n}=19,2$ females, 17 males, ages 17-63 months), regressive autism (AU-reg; $\mathrm{n}=17,3$ females, 14 males, ages 28-60 months), a control group of agematched typically developing children (TD; $n=28,10$ females, 18 males, ages 17-120 months) and children with developmental disabilities (DD) without autism ( $\mathrm{n}=16 ; 5 \mathrm{~F}, 11 \mathrm{M}$, ages 12-54 months).

All children were assessed at the UC Davis M.I.N.D. Institute. Autism was confirmed using the Autism Diagnostic Interview-Revised (ADI-R ${ }^{[28,29]}$ and the Autism Diagnostic Observation Schedule, modules 1,2 and 3 (ADOS) $\left({ }^{[30-32]}\right)$. The ADI-R is a standardized semi-structured interview that provides a diagnostic algorithm for the DSM-IV ${ }^{[33]}$ and the ICD-10 definitions of autism ${ }^{[34,}{ }^{35]}$. The ADOS is a standardized, semi-structured assessment in which the researcher observes the social behavior, communication and imaginative use of materials for children suspected of having ASD. A diagnosis of autism was defined as meeting criteria on the communication, social and repetitive behaviors domains of the ADI-R and scoring at or above the cut off for autistic disorder on the ADOS module 1 or 2.

The Social Communication Questionnaire was used to screen for behavioral and developmental characteristics of ASD among subjects with developmental disabilities and typically developing controls. Children who scored above the screening cutoff were fully assessed using the ADI-R and ADOS.

The children with autism were further subdivided into children who initially developed normally, reaching typical developmental milestones before regressing and losing language and social skills and those who had early impairments in the development of language and social skills. A classification of regression was based on clinical characteristics using both parental reporting and answers to questions regarding language loss (Q11) and social skills (Q25) of the ADI-R. Our autism study population could be classified into 19 subjects with early onset autism (classical) and 17 subjects with delayed-onset autism (regression). The study protocol followed the ethical guidelines of the most recent Declaration of Helsinki and was approved by the Institutional Review Boards of the UC Davis School of Medicine and the State of California and all subjects enrolled in the study had written informed consent provided by their parents and assented to participate if developmentally able.

Blood samples were collected into yellow top citrate-containing tubes and plasma was isolated by centrifugation at $2300 \mathrm{rpm}$ for 10 minutes. Plasma aliquots were stored at $-80^{\circ} \mathrm{C}$ until use.

Levels of $\beta$-2-microglobulin were measured using ELISA kits (Bioquant) according to the manufacturer's protocols. Briefly, samples were diluted 1:100 in the provided diluent. $20 \mu \mathrm{L}$ of sample or standard and 200 $\mu \mathrm{L}$ of diluent were pipetted in duplicate into the appropriate pre-coated wells and incubated at $37^{\circ} \mathrm{C}$ for 30 minutes. Plates were washed 5 times before addition of $200 \mu \mathrm{L}$ of enzyme conjugate reagent. Following a 30-minute incubation, the wash step was repeated and $100 \mu \mathrm{L}$ of TMB was added. After 20 minutes, a stop solution was added to the wells and absorbance was read at $450 \mathrm{~nm}$. Concentrations were calculated using a standard curve plotting absorbance versus standard concentration. Sample concentrations were calculated with the equation derived from the curve. Statistical differences between groups were determined using Student's two-tailed t-test for samples with unequal variances. Values were considered significant if $p$ was less than 0.05 .

\section{RESULTS AND DISCUSSION}

We found no statistically significant differences in $\beta$-2-microglobulin levels between AU (median value: $1.071 \mu \mathrm{g} / \mathrm{ml}$, interquartile range: $0.830-1.311$ ), age matched DD (1.312, 0.793-1.943) and age matched TD controls (0.952, 0.826-1.387) (Fig. 1a). Based on autism onset patterns, we further divided the AU group into early onset (EO) $(0.908,0.722-1.509)$ and regressive $(0.841,0.718-1.322)$ phenotypes ${ }^{[26]}$ and similarly found no difference between groups (Fig. 1b).

The current study explores a possible connection between ASD and MHC I expression as represented by serum levels of $\beta$-2-microglobulin ( $(32 \mathrm{~m})$. We chose to investigate this potential link in response to research suggesting that neuronal MHC I expression is required for proper neurodevelopment and synaptic plasticity (reviewed in ${ }^{[18,19]}$ ).

For years it was thought that neuronal cells did not express MHC I under normal conditions. This was one 


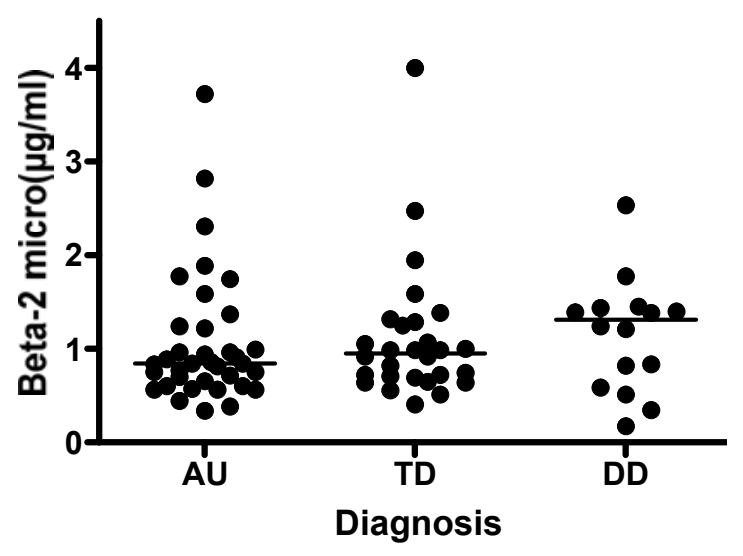

Fig. 1a: Beta-2-microglobulin levels in AU, TD and DD subjects (A) Scatter plot representing levels of B-2-microglobulin in subjects with AU compared to TD and DD, bars represent median values

of many factors believed to contribute to the "immuneprivileged" status of the brain. However, several studies have now shown that MHC I is in fact expressed by healthy neurons ${ }^{[21,22,36]}$. Furthermore, MHC I has several non-immunological roles and is involved in several aspects of neuronal development and function. First, MHC I gene expression levels have been linked to activity-dependent plasticity in the visual system ${ }^{[21]}$. This finding was corroborated in a study with MHC I deficient mice that demonstrated the necessity of MHC I for the development of visual projections and longterm potentiation in the adult hippocampus ${ }^{[22]}$. Additionally, a 2004 study showed that MHC I was vital for the selective maintenance of neuronal synapses after induction of axonal lesions ${ }^{[23]}$. Together, these studies suggest that low levels of neuronal MHC I expression may contribute to a loss of synaptic plasticity and axon regeneration.

In addition to its involvement in synaptic maintenance in the brain, MHC I has also been linked to behavioral traits ${ }^{[37]}$. MHC I molecules have been shown to associate with certain pheromone receptors and aid their expression on the surface of neurons in the vomeronasal organ in mice ${ }^{[38]}$. $\beta 2 \mathrm{~m}$ deficient mice showed decreased expression of certain pheromone receptors and exhibit decreased male-male $\operatorname{aggression}^{[38]}$.

Subjects with ASD have abnormal brain circuitry, which may include high local synaptic connectivity and low long-range synaptic connectivity ${ }^{[39]}$. Abnormalities in synaptic and columnar structure, as well as increased

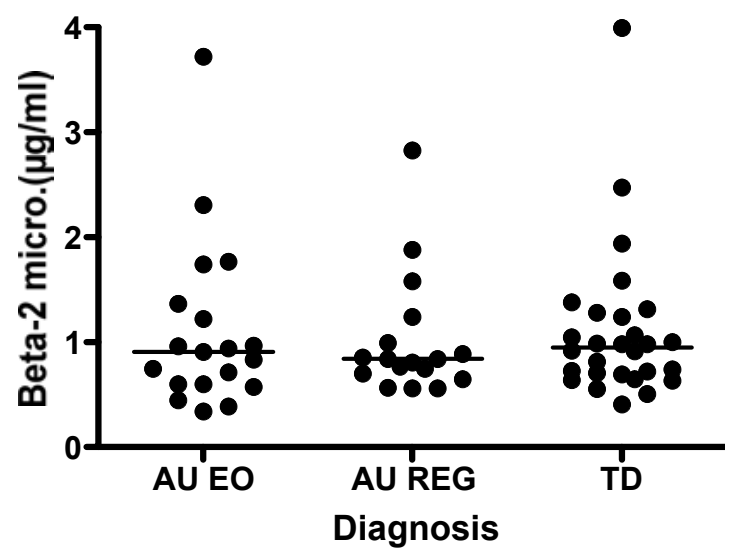

Fig. 1b: Comparison of $\beta-2-$ microglobulin levels in children with early onset (EO or classic) autism, regressive (reg) autism and TD controls

cell density in the cerebral cortex have also been demonstrated in individuals with $\mathrm{ASD}^{[40-44]}$. In addition, children with ASD have been shown to have larger brain volumes than typically developing children ${ }^{[45]}$. MHC I deficient mice exhibit many similar structural features in several brain regions ${ }^{[22]}$. Thus, in addition to genetic linkages, the involvement of MHC I in brain formation and behavior make it an ideal candidate to study in relation to ASD.

In our cross-sectional analysis, we found there to be no significant difference in plasma $32 \mathrm{~m}$ between subjects with ASD and TD controls. However, the negative results reported herein do not exclude the possibility that MHC I may play a role in ASD development. First, due to the clinical nature of our study, it was not possible to directly measure the expression of MHC I by neuronal cells. It is possible that serum $\beta 2 \mathrm{~m}$ levels are not fully representative of $\mathrm{MHC}$ expression in the brain. Perhaps a better source would be cerebral spinal fluid (CSF). Second, MHC I expression on neurons is developmentally regulated and is highest during perinatal periods ${ }^{[21,22]}$. A longitudinal study encompassing several stages of development may render more accurate results and future research should explore this possibility.

\section{ACKNOWLEDGEMENTS}

NIEHS 1 P01 ES11269-01, the U.S. Environmental Protection Agency (U.S. EPA) through the Science to Achieve Results (STAR) program (Grant R829388) and the UC Davis M.I.N.D Institute, supported this work. 


\section{REFERENCES}

1. Lord, C. et al., 2000. The autism diagnostic observation schedule-generic: a standard measure of social and communication deficits associated with the spectrum of autism. J. Autism. Dev. Disord., 30: 205-23.

2. Lord, C. et al., 2000. Autism spectrum disorders. Neuron, 28: 355-63.

3. Anonymous, 2007. Prevalence of autism spectrum disorders--autism and developmental disabilities monitoring network, 14 sites, United States, 2002. MMWR Surveill. Summ., 56: 12-28.

4. Ashwood, P., S. Wills and J. Van de Water, 2006. The immune response in autism: a new frontier for autism research. J. Leukoc. Biol., 80: 1-15.

5. Vargas, D.L. et al., 2005. Neuroglial activation and neuroinflammation in the brain of patients with autism. Ann. Neurol., 57: 67-81.

6. Croonenberghs, J. et al., 2002. Activation of the inflammatory response system in autism. Neuropsychobiology, 45: 1-6.

7. Ashwood, P. and J. Van de Water, 2004. Is autism an autoimmune disease? Autoimmun. Rev., 3: 55762.

8. Connolly, A.M. et al., 1999. Serum autoantibodies to brain in Landau-Kleffner variant, autism and other neurologic disorders. J. Pediatr, 134: 607-13.

9. Connolly, A.M. et al., 2005. Brain-Derived Neurotrophic Factor and Autoantibodies to Neural Antigens in Sera of Children with Autistic Spectrum Disorders, Landau-Kleffner Syndrome and Epilepsy. Biol Psychiatry.

10. Zimmerman, A.W. et al., 2007. Maternal antibrain antibodies in autism. Brain Behav. Immun., 21: 351-357.

11. Singer, H.S. et al., 2006. Antibrain antibodies in children with autism and their unaffected siblings. J. Neuroimmunol., 178: 149-55.

12. Patterson, P.H., 2002. Maternal infection: window on neuroimmune interactions in fetal brain development and mental illness. Curr. Opin. Neurobiol., 12: 115-8.

13. Fatemi, S.H. et al., 2002. Prenatal viral infection leads to pyramidal cell atrophy and macrocephaly in adulthood: implications for genesis of autism and schizophrenia. Cell Mol. Neurobiol., 22: 2533.

14. Libbey, J.E. et al., 2005. Autistic disorder and viral infections. J. Neurovirol., 11: 1-10.
15. Warren, R.P. et al., 1991. Increased frequency of the null allele at the complement $\mathrm{C} 4 \mathrm{~b}$ locus in autism. Clin. Exp. Immunol., 83: 438-40.

16. Daniels, W.W. et al., 1995. Increased frequency of the extended or ancestral haplotype B44-SC30DR4 in autism. Neuropsychobiology, 32: 120-3.

17. Mehler, M.F. and J.A. Kessler, 1998. Cytokines in brain development and function. Adv. Protein Chem., 52: 223-51.

18. Boulanger, L.M. and C.J. Shatz, 2004. Immune signalling in neural development, synaptic plasticity and disease. Nat. Rev. Neurosci., 5: 52131.

19. Boulanger, L.M., G.S. Huh and C.J. Shatz, 2001. Neuronal plasticity and cellular immunity: shared molecular mechanisms. Curr. Opin. Neurobiol., 11: 568-78.

20. Olson, R., C. Dulac and P.J. Bjorkman, 2006. MHC homologs in the nervous system--they haven't lost their groove. Curr. Opin. Neurobiol., 16: 351-7.

21. Corriveau, R.A., G.S. Huh and C.J. Shatz, 1998. Regulation of class I MHC gene expression in the developing and mature CNS by neural activity. Neuron, 21: 505-20.

22. Huh, G.S. et al., 2000. Functional requirement for class I MHC in CNS development and plasticity. Science, 290: 2155-9.

23. Oliveira, A.L. et al., 2004. A role for MHC class I molecules in synaptic plasticity and regeneration of neurons after axotomy. Proc. Natl. Acad. Sci. USA, 101: 17843-8.

24. Wright, P. et al., 2001. Schizophrenia and HLA: A review. Schizophr. Res., 47: 1-12.

25. Tsuchiya, N. et al., 1996. Elevated serum level of soluble HLA class I antigens in patients with systemic lupus erythematosus. Arthritis Rheum., 39: 792-6.

26. Rogers, S.J., 2004. Developmental regression in autism spectrum disorders. Ment. Retard. Dev. Disabil. Res. Rev., 10: 139-43.

27. Hertz-Picciotto, I. et al., 2006. The CHARGE study: an epidemiologic investigation of genetic and environmental factors contributing to autism. Environ. Health Perspect., 114: 1119-25.

28. Lord, C. et al., 1997. Diagnosing autism: analyses of data from the Autism Diagnostic Interview. J. Autism. Dev. Disord., 27: 501-17.

29. Lord, C., M. Rutter and A. Le Couteur, 1994. Autism Diagnostic Interview-Revised: a revised version of a diagnostic interview for caregivers of individuals with possible pervasive developmental disorders. J. Autism. Dev. Disord., 24: 659-85. 
30. Lord, C., B.L. Leventhal and E.H. Cook, Jr., 2001. Quantifying the phenotype in autism spectrum disorders. Am. J. Med. Genet., 105: 36-8.

31. Joseph, R.M., H. Tager-Flusberg and C. Lord, 2002. Cognitive profiles and social-communicative functioning in children with autism spectrum disorder. J. Child Psychol. Psychiatry, 43: 807-21.

32. DiLavore, P.C., C. Lord and M. Rutter, 1995. The pre-linguistic autism diagnostic observation schedule. J. Autism. Dev. Disord., 25: 355-79.

33. Association, A.P., Diagnostic and Statistical Manual of Mental Disorders, Fourth Edition. 1994, Washington, DC: American Psychiatric Association.

34. Bebbington, P., 1992. Welcome to ICD-10. Soc Psychiatry Psychiatr Epidemiol, 27: 255-7.

35. Steinhausen, H.C. and A. Erdin, 1992. Abnormal psychosocial situations and ICD-10 diagnoses in children and adolescents attending a psychiatric service. J. Child Psychol. Psychiatry, 33: 731-40.

36. Lidman, O., T. Olsson and F. Piehl, 1999. Expression of nonclassical MHC class I (RT1-U) in certain neuronal populations of the central nervous system. Eur. J. Neurosci., 11: 4468-72.

37. Dulac, C. and A.T. Torello, 2003. Molecular detection of pheromone signals in mammals: from genes to behaviour. Nat. Rev. Neurosci., 4: 551-62.
38. Loconto, J. et al., 2003. Functional expression of murine $\mathrm{V} 2 \mathrm{R}$ pheromone receptors involves selective association with the M10 and M1 families of MHC class Ib molecules. Cell, 11: 607-18.

39. Just, M.A. et al., 2004. Cortical activation and synchronization during sentence comprehension in high-functioning autism: Evidence of underconnectivity. Brain, 127: 1811-21.

40. Williams, R.S. et al., 1980. Autism and mental retardation: neuropathologic studies performed in four retarded persons with autistic behavior. Arch. Neurol., 37: 749-53.

41. Casanova, M.F., D.P. Buxhoeveden and C. Brown, 2002. Clinical and macroscopic correlates of minicolumnar pathology in autism. J. Child Neurol., 17: 692-5.

42. Casanova, M.F. et al., 2002. Neuronal density and architecture (Gray Level Index) in the brains of autistic patients. J. Child Neurol., 17: 515-21.

43. Casanova, M.F. et al., 2002. Asperger's syndrome and cortical neuropathology. J. Child Neurol., 17: 142-5.

44. Casanova, M.F. et al., 2002. Minicolumnar pathology in autism. Neurology, 58: 428-32.

45. Courchesne, E., R. Carper and N. Akshoomoff, 2003. Evidence of brain overgrowth in the first year of life in autism. JAMA, 290: 337-44. 